Check for updates

Cite this: Chem. Sci., 2018, 9, 6975

๑ All publication charges for this article have been paid for by the Royal Society of Chemistry

Received 25th January 2018

Accepted 19th July 2018

DOI: $10.1039 / c 8 s c 00406 d$

rsc.li/chemical-science

\title{
Rapid, non-invasive characterization of the dispersity of emulsions via microwaves $\uparrow$
}

\author{
Yuqiang Yan, (D) ab Sergio Gonzalez-Cortes, (D) ${ }^{\mathrm{b}}$ Benzhen Yao, (D) ${ }^{\mathrm{b}}$ \\ Daniel R. Slocombe, (D) ${ }^{c}$ Adrian Porch, (D) ${ }^{c}$ Fahai Cao, (D)*a Tiancun Xiao (iD ${ }^{b}$ \\ and Peter P. Edwards (D)*b
}

\begin{abstract}
A rapid and non-invasive method to determine the dispersity of emulsions is developed based on the interrelationship between the droplet size distribution and the dielectric properties of emulsions. A range of water-in-oil emulsions with different water contents and droplet size distributions were analysed using a microwave cavity perturbation technique together with dynamic light scattering. The results demonstrate that the dielectric properties, as measured by non-invasive microwave cavity analysis, can be used to characterise the dispersity of emulsions, and is also capable of characterizing heavy oil emulsions. This technique has great potential for industrial applications to examine the sedimentation, creaming and hence the stability of emulsions.
\end{abstract}

\section{Introduction}

An emulsion is a mixture of two or more immiscible liquids with one liquid dispersed in the other. Water-in-oil (W/O) emulsions are widely used in polymerization, ${ }^{1}$ oil refinery, ${ }^{2,3}$ pharmaceuticals, ${ }^{4}$ cosmetic ${ }^{5}$ and food industries. ${ }^{6}$ As an emulsion is thermodynamically unstable, it is inevitably polydispersed to some degree. ${ }^{7}$ The important droplet size distribution describes the emulsion dispersity, which influences a range of important characteristics such as emulsion stability, viscosity and rheological behaviour.

Common methods for determining the droplet size distribution of common emulsions include microscopy, ultrasonics and light scattering techniques, ${ }^{\mathbf{8} 9}$ but these methods are limited to opaque samples like crude oil emulsions in oil refinery and petrochemical industries. The presence of gas bubbles and suspended solids also considerably restrict their applicability to such emulsion systems. ${ }^{10}$

Much effort has been made recently to explore alternative methods for determining the droplet size of emulsions, including Nuclear Magnetic Resonance (NMR), ${ }^{\mathbf{1 1}, 12}$ Raman spectroscopy ${ }^{13}$ and confocal laser scanning microscope. ${ }^{\mathbf{1 4}}$ However, the measurement error of above techniques is

${ }^{a}$ School of Chemical Engineering, East China University of Science and Technology, Shanghai 200237, China. E-mail: fhcao@ecust.edu.cn

${ }^{b}$ King Abdulaziz City for Science and Technology-Oxford Centre of Excellence for Petrochemicals(KOPRC), Inorganic Chemistry Laboratory, Department of Chemistry, University of Oxford, Oxford OX1 3QR, UK. E-mail: peter.edwards@chem.ox.ac.uk ${ }^{c}$ School of Engineering, Cardiff University, Queen's Buildings, The Parade, Cardiff CF24 3AA, UK

† Electronic supplementary information (ESI) available. See DOI: 10.1039/c8sc00406d challenged especially at low water content. Therefore, it is of considerable scientific and also industrial significance to develop an easy, rapid and more accurate way to assess the dispersity of emulsions.

An alternative rapid and non-invasive method to characterise the dispersity of emulsions is reported here based on the interrelationship between the droplet size distribution and the characteristic dielectric properties of emulsions. An effective mixing model is proposed to correlate the dielectric constant of the emulsion system with the emulsion dispersity. For this, we have investigated the dielectric properties of emulsified oil having different water contents and droplet size distributions using a microwave cavity perturbation technique. The applicability of this technique for heavy oil emulsions is also demonstrated.

\section{Theoretical considerations}

The dielectric properties of emulsions cannot be simply derived from the properties of the individual pure components due to the existence of interfacial polarization and local field corrections. ${ }^{15}$ Several theories, such as those of Maxwell, ${ }^{16}$ Wagner, ${ }^{17}$ Hanai $^{15}$ and Lichtenecker, ${ }^{18}$ have been reported in the last century to estimate the dielectric properties of mixtures and thereafter used to predict or rationalise the dielectric properties of emulsions. ${ }^{19,20}$ However, these models are all based on the assumption that the dispersed phase is considered as assembly identical spheres in the continuous phase. In fact, a size distribution of dispersed droplets will always exist in a real emulsion system, and previous studies demonstrated that the size distribution of micelles influences the permittivity of the mixture. ${ }^{21}$ Therefore, it is essential to take carefully into account 
the dispersity of emulsion in any mixing model for dielectric properties.

A monodispersed water-in-oil emulsion system, in which all the water droplets are assumed to be identical spheres, is illustrated in Fig. 1a. The dielectric constant of this system can be readily estimated by Lichtenecker theory ${ }^{18,22}$

$$
\ln \varepsilon_{\mathrm{m}}=\varphi_{\mathrm{d}} \ln \varepsilon_{\mathrm{d}}+\left(1-\varphi_{\mathrm{d}}\right) \ln \varepsilon_{\mathrm{c}}
$$

where $\varepsilon_{\mathrm{m}}$ is the dielectric constant of the emulsion, $\varphi_{\mathrm{d}}$ is the volume fraction of the dispersed water phase, and $\varepsilon_{\mathrm{d}}$ and $\varepsilon_{\mathrm{c}}$ are the dielectric constant of the water and continuous oil phase respectively.

When the two phases are fully phase-separated with the interface perpendicular to the orientation of the electric field, as is shown in Fig. 1b, this system can be considered as two serial capacitors, thus the dielectric constant in this case is given by

$$
\frac{1}{\varepsilon_{\mathrm{m}}}=\frac{\varphi_{\mathrm{d}}}{\varepsilon_{\mathrm{d}}}+\frac{1-\varphi_{\mathrm{d}}}{\varepsilon_{\mathrm{c}}}
$$

Here we have proposed an effective physical system which is equivalent to the real emulsion system (Fig. 1c), since a real emulsion could be regarded as a transition state from the monodispersed phase (Fig. 1a) to the separated phase (Fig. 1b). As illustrated in Fig. 1d, identical water drops are uniformly dispersed in the upper oil phase of this system, and the lower phase is water phase settled at the bottom. The dielectric constant of this system can be presented as

$$
\varepsilon_{\mathrm{e}}=\frac{\varepsilon_{\mathrm{d}} \varepsilon_{\mathrm{m}}}{\varphi_{\mathrm{w} 2} \varepsilon_{\mathrm{m}}+\left(1-\varphi_{\mathrm{w} 2}\right) \varepsilon_{\mathrm{d}}}
$$

where $\varepsilon_{\mathrm{e}}$ is the effective dielectric constant of the emulsion system, $\varphi_{\mathrm{w} 2}$ is the volume fraction of the lower water phase, and
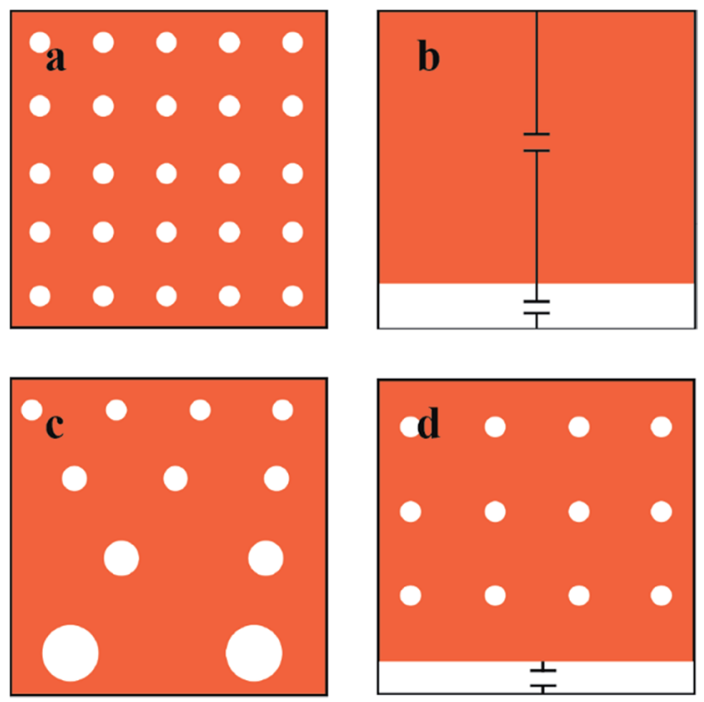

Fig. 1 Schematic diagrams of emulsion systems. (a) Monodispersed emulsion. (b) Phase separation (regarded as two serial capacitors). (c) Polydispersed emulsion. (d) Effective mixing model. The continuous phase (in yellow colour) is the oil phase, while the dispersed phase (in white colour) is the water phase. $\varepsilon_{\mathrm{m}}$ is the dielectric constant of the upper phase which can be obtained by

$$
\ln \varepsilon_{\mathrm{m}}=\varphi_{\mathrm{w} 1} \ln \varepsilon_{\mathrm{d}}+\left(1-\varphi_{\mathrm{w} 1}\right) \ln \varepsilon_{\mathrm{c}}
$$

where $\varphi_{\mathrm{w} 1}$ is the volume fraction of the water at the upper phase in the whole volume.

Here a definition of dispersity factor $D$ is given by

$$
D=\frac{\varphi_{\mathrm{w} 1}}{\varphi_{\mathrm{w} 1}+\varphi_{\mathrm{w} 2}}
$$

For a known emulsion composition, the dispersity factor $D$ can be determined from the measured dielectric constants. The value of $D$ is between 0 and 1 , which can be understood as the degree of polydispersity of the water droplets. $D=1$ denotes a monodispersed emulsion, while $D=0$ suggests that the system has been fully separated into two phases. Hence, we can use the dispersity factor to describe the dispersity of real emulsion systems.

\section{Results and discussion}

\section{Experimental setup}

The dielectric properties of emulsions were measured via a microwave cavity perturbation measurement. The microwave cavity perturbation measurement system was fabricated in Inorganic Chemistry Laboratory at University of Oxford. The cavity (Fig. 2a) is made of aluminium and in a cylindrical shape. The cavity excitation is provided by two open-circuit SMA connectors coupled into the electric field at a frequency of 2.5 GHz. The $\mathrm{TM}_{010}$ mode is employed to establish a highly uniform E-field near the axis, resulting in a minimal depolarization of the sample in the measurement. ${ }^{23}$ The electric field distribution in this cavity simulated by COMSOL Multiphysics is shown in Fig. 2b. In the measurement, the sample was loaded into a quartz tube and placed in the centre of the cavity, since the inner tube radius $(2 \mathrm{~mm})$ is far smaller than the radius of cavity, the E-field applied to the sample can be regarded highly uniform.

The measurements were performed using an Agilent E5071B network analyser by which the transmitted power $\left|S_{21}\right|^{2}$ was measured in the frequency domain, then we used non-linear
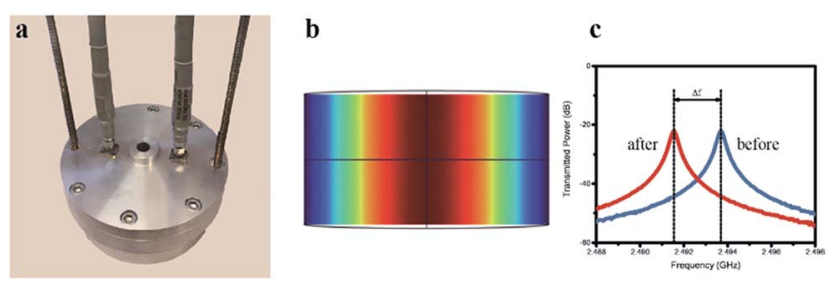

Fig. 2 The microwave cavity perturbation measurement system. (a) The microwave cavity. The inner radius and length of the cavity are $4.6 \mathrm{~cm}$ and $4.0 \mathrm{~cm}$ respectively (b) the electric field distribution inside the cavity. The E-field antinode is near the cavity axis (in red colour). (c) Transmitted power plot before and after loading the sample. 
least-square fitting to determine the resonant frequency $f$ and the loaded quality factor $Q$. Loading the sample into the cavity causes a negative shift in the resonant frequency $f$ (Fig. 2c) as a result of the sample polarization in response to the microwave electric field, thus we can derive the dielectric constant $\varepsilon$ of emulsions from the variation in resonant frequency $\Delta f$ using first order perturbation theory ${ }^{24}$

$$
(\varepsilon-1) A V_{\mathrm{s}} \cong \frac{\Delta f}{f_{0}}
$$

where $A$ is a constant that depends on the cavity geometry and size, and $A=7.11 \times 10^{-3} \mathrm{~cm}^{-3}$ for this system which was determined by a polytetrafluoroethylene (PTFE) sample of known permittivity. $f_{0}$ is the frequency for the cavity with an empty tube, $V_{\mathrm{s}}$ is the sample volume in the cavity and $V_{\mathrm{s}}=$ $0.126 \mathrm{~cm}^{3}$ in the measurements. Each sample has been measured three times, and the dispersity factor of the sample was calculated from the average measured dielectric constant.

In this work, we measured the dielectric properties of emulsions using the microwave cavity perturbation technique. The attractive features of this approach are as follows: (1) it is a rapid measurement which takes only milliseconds, thus allowing this application to real time analysis and control; (2) instead of focusing on a single point, the microwaves penetrate the entire sample based on the sample holder dimension. Therefore, a reliable volumetric analysis of emulsions can be determined; (3) the microwave power level in the measurements is $0 \mathrm{dBm}$ (i.e. $1 \mathrm{~mW}$ per $\mathrm{rms}$ ), so there is negligible sample heating. We note that this technique is non-invasive and nondestructive, thereby providing a potential means of a rapid in situ industrial analysis.

\section{Deviation from values predicted by theoretical models}

Initially we measured the dielectric properties of emulsions with different water contents (0-20.0 vol\%) and compared the data with the values predicted by the mixing models. As shown in Fig. 3, the dielectric constant of water-in-oil emulsions increases rapidly with the water content, because the dielectric constant of water is much higher than the value of oil phase. The sensitivity to the water content offers potential applications of this technique for multiphase flow meters. ${ }^{25}$ MATLAB R2017a was employed to calculate the dielectric constant of emulsions from the mixing models. The results show that for a certain water content, the values predicted by Lichtenecker model are higher than the values calculated from serial capacitor model. The deviation between the measured dielectric constant and the two limiting predicted values arises from the polydispersity of the water phase, thus we can directly investigate the dispersity of emulsions through the variation of measured dielectric constant.

Here we chose dodecane and hexadecane as model oils since their emulsions are transparent and can be accurately interrogated and analysed by the dynamic light scattering (DLS) technique. The dielectric constants as well as the droplet size distribution of water-in-dodecane emulsions for different emulsifying time were investigated using the microwave cavity
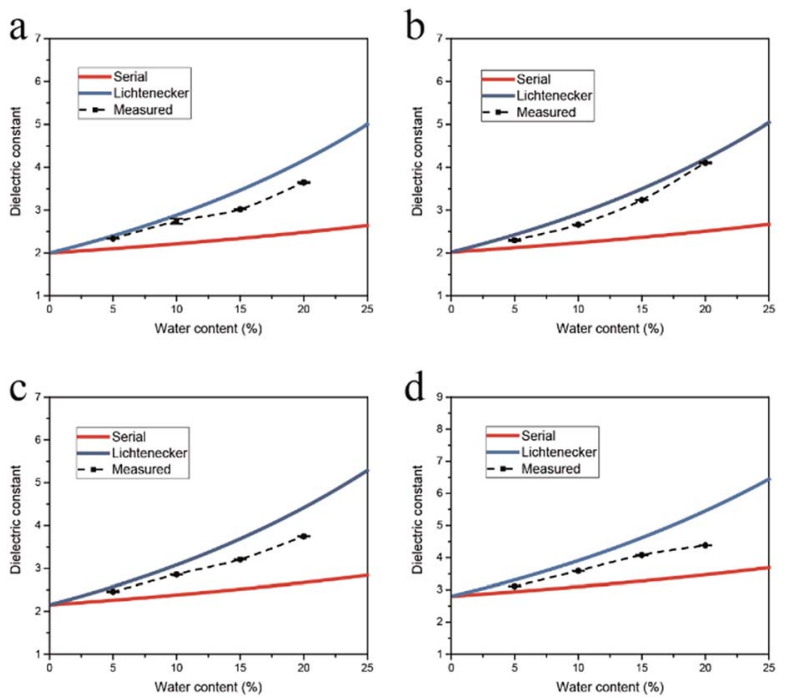

Fig. 3 Dielectric constant of emulsions with different water contents. (a) Water-in-dodecane emulsions. (b) Water-in-hexadecane emulsions. (c) Water-in-crude oil emulsions. (d) Water-in-tar oil emulsions. Emulsifications were performed at a rotation speed of $10000 \mathrm{rpm}$ and emulsifying time of $10 \mathrm{~min}$. The blue line denotes the dielectric constant calculated by serial capacitor model, and the red line denotes the values predicted by Lichtenecker model.

analysis and DLS. As shown in Fig. 4a, the measured dielectric constant is near the value predicted by serial capacitor model at emulsifying time of 1 min, suggesting that the emulsion system is non-uniform. Then the dielectric constant has a rising trend with the emulsifying time, and correspondingly the dispersity factor increases from 0.04 at $1 \mathrm{~min}$ to 0.66 at $20 \mathrm{~min}$ (Fig. 4b).

\section{The variation of dispersity factors is consistent with the DLS measurement results}

The droplet size distributions obtained by DLS are illustrated in Fig. 4c. The multiple peaks at $1 \mathrm{~min}$ indicate a broad size distribution of water droplets. With an increase in the emulsifying time, the distribution peaks have a negative shift and a narrower bandwidth, demonstrating the emulsion system is more uniform. According to energy dissipation theory, the droplet size distribution for emulsification in the turbulent regime is determined by the energy density, dimensionally related to the residence time, the rotator speed and diameter. ${ }^{26,27}$ The average droplet size of dispersed phase should decrease with the increase of the emulsifying time, which is consistent with the measured DLS results.

The measurements were also performed on a series of waterin-hexadecane emulsion samples which were prepared for different emulsifying time. As shown in Fig. $5 a$ and b, though the water content of emulsions is rather low $(2.0 \mathrm{vol} \%)$, the measured dielectric constant increases considerably with the emulsifying time increasing, and the dispersity factor rises from 0.03 at $5 \mathrm{~min}$ to 0.89 at $15 \mathrm{~min}$. The size distribution determined by DLS verified that the polydispersity of samples reduces with the emulsifying time (Fig. 5c). 

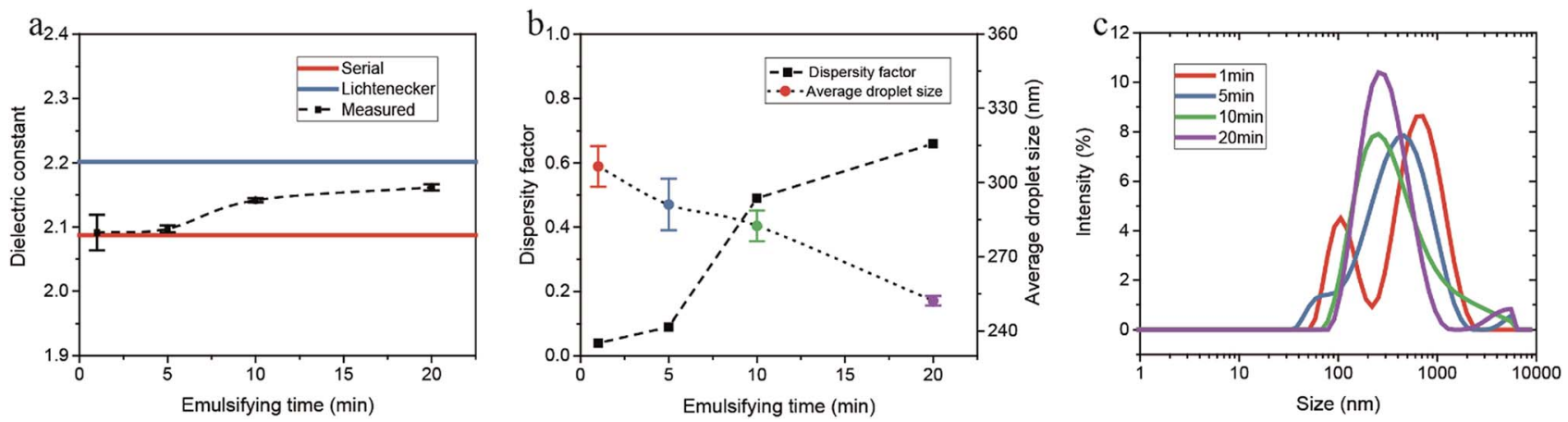

Fig. 4 The measured data and calculation results for the water-in-dodecane emulsions prepared for different emulsifying time. (a) The dielectric constant. (b) The dispersity factors and the average droplet size (measured by DLS). (c) The normalized droplet size distribution. The water content is $2.0 \mathrm{vol} \%$ and emulsifications were performed at a rotation speed of $10000 \mathrm{rpm}$.
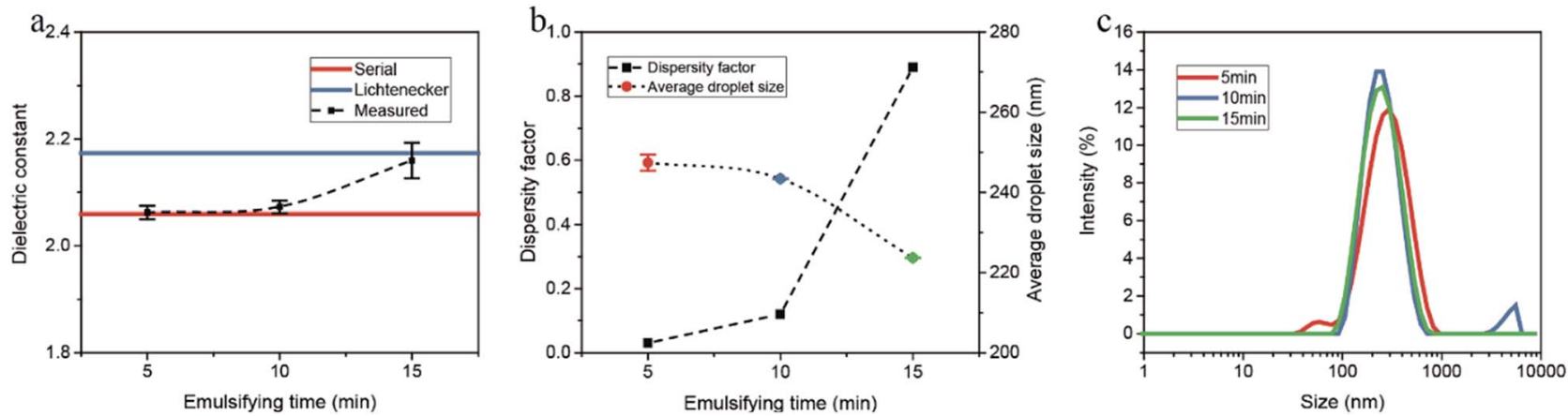

Fig. 5 The measured data and calculation results for the water-in-hexadecane emulsions prepared for different emulsifying time. (a) The dielectric constant. (b) The dispersity factors and the average droplet size (measured by DLS). (c) The normalized droplet size distribution. The water content is 2.0 vol\% and emulsifications were performed at a rotation speed of $10000 \mathrm{rpm}$.

The droplet size distribution of emulsions is influenced significantly by the rotation speed during emulsion preparation. We conducted both the microwave cavity analysis and DLS on water-in-dodecane emulsions prepared with different rotation speeds, and the measured data and calculation results are shown in Fig. 6. The dielectric constant of emulsions slightly increases with the emulsifying rotation speed, and correspondingly the dispersity factor rises from 0.37 at $10000 \mathrm{rpm}$ to 0.88 at $16000 \mathrm{rpm}$. The energy density for emulsification increases with rotation speed, thus resulting in a smaller average droplet size. We note that the emulsions have a narrower distribution at a higher rotation speed, as illustrated in DLS size curves (Fig. 6c).

We also analysed a series of water-in-hexadecane emulsion samples prepared with various rotation speeds. As illustrated in Fig. 7, the dielectric constant as well as the dispersity factor rises with increasing rotation speed. The droplet distributions measured by DLS are consistent with the variation of the dielectric constant.
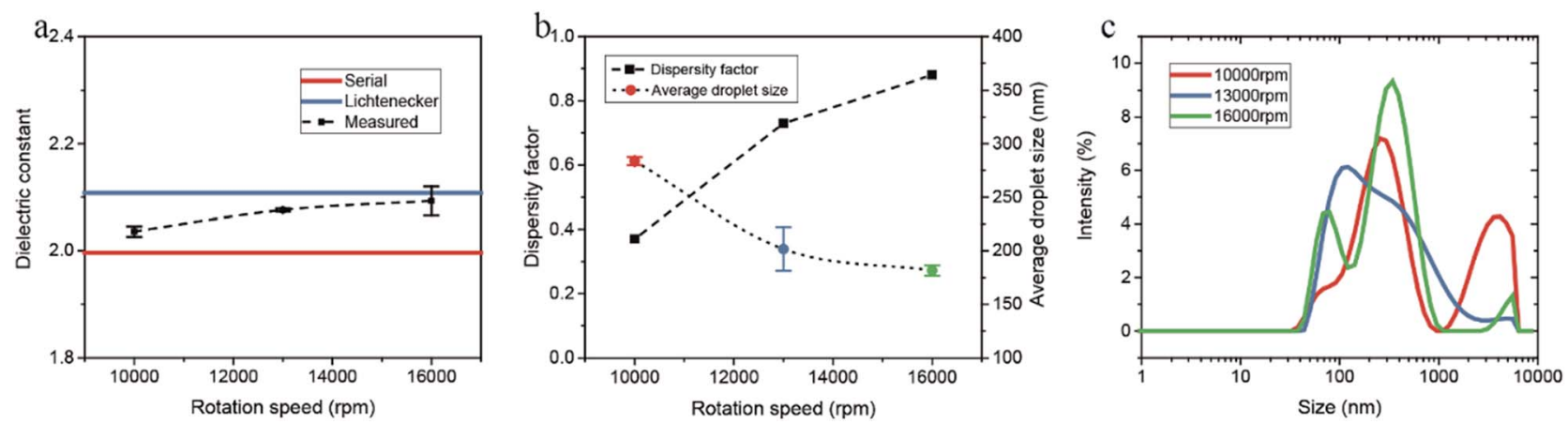

Fig. 6 The measured data and calculation results for the water-in-dodecane emulsions with different rotation speed. (a) The dielectric constant. (b) The dispersity factors and the average droplet size (measured by DLS). (c) The normalized droplet size distribution. The water content is 2.0 vol\% and emulsifications were performed for an emulsifying time of $2 \mathrm{~min}$. 

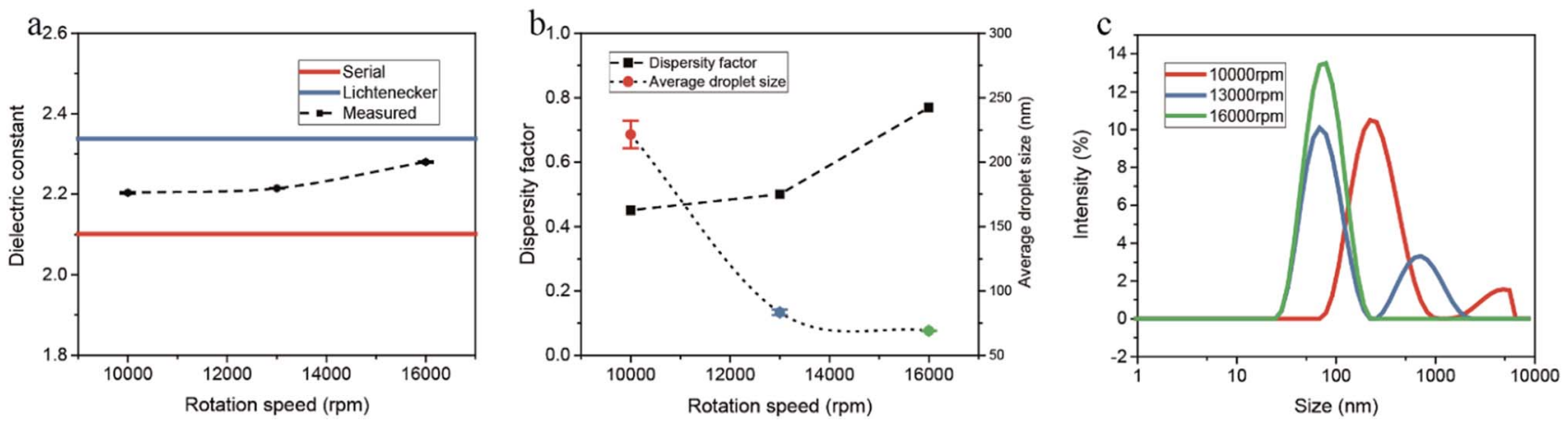

Fig. 7 The measured data and calculation results for the water-in-hexadecane emulsions with different rotation speed. (a) The dielectric constant. (b) The dispersity factors and the average droplet size (measured by DLS). (c) The normalized droplet size distribution. The water content is 2.0 vol\% and emulsifications were performed for an emulsifying time of 2 min.

Therefore, we can verify that the dispersity factor most effectively describes the size distribution of dispersed droplets. A higher dispersity factor indicates a narrower droplet distribution and hence a more uniform emulsion. Importantly, the microwave cavity analysis is critically sensitive to the variation of droplet size distribution even at low water content due to the significant difference between the dielectric constant of water and oil. Although these measurements have been conducted at the ISM frequency of $2.45 \mathrm{GHz}$, it will also be possible to perform similar measurements on inhomogeneous mixtures of greater volume by increasing (proportionately) the size of the cavity, thus reducing the resonant frequency of the $\mathrm{TM}_{010}$ mode. One such frequency within an ISM band is around $900 \mathrm{MHz}$, for which the host cavity will have an internal diameter of around $25.5 \mathrm{~cm}$. We have found in other work that it is possible to have a sample tube with an internal diameter of up to $5 \mathrm{~cm}$, with a pair of axial chokes, thus maintaining a very high $Q$ factor. For such a $900 \mathrm{MHz}$ cylindrical cavity of aspect ratio diameter to height of $1: 1$, this yields an active sample volume of up to 0.5 litres.

\section{Employed for emulsified heavy oil characterization}

We also conducted the microwave cavity analysis on heavy fossil oil emulsions. Compared to paraffins, both the crude oil and tar oil have a rather complex composition, containing
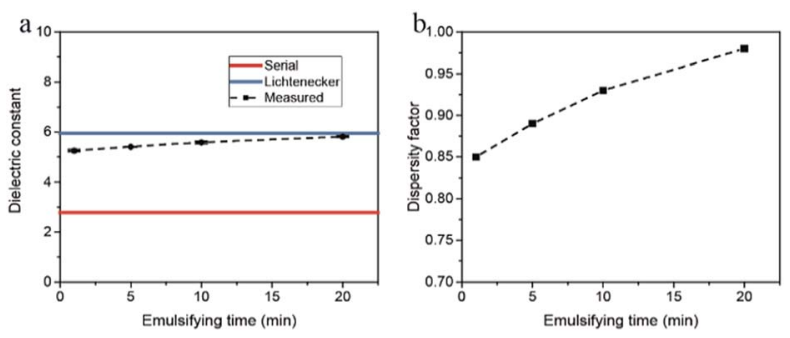

Fig. 8 The measured data and calculation results for the water-incrude oil emulsions prepared for different emulsifying time. (a) The dielectric constant. (b) The dispersity factors. The water content is $10.0 \mathrm{vol} \%$ and emulsifications were performed at a rotation speed of $10000 \mathrm{rpm}$.
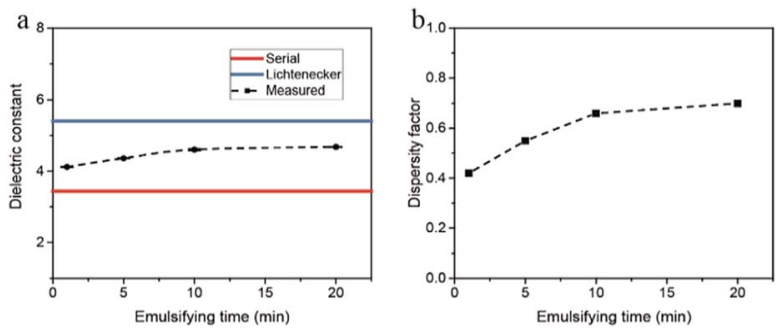

Fig. 9 The measured data and calculation results for the water-in-tar oil emulsions prepared for different emulsifying time. (a) The dielectric constant. (b) The dispersity factors. The water content is $10.0 \mathrm{vol} \%$ and emulsifications were performed at a rotation speed of $10000 \mathrm{rpm}$.

hundreds of compounds. Due to the opaqueness of emulsified oil, one cannot measure the droplet size distribution accurately using dynamic light scattering technique. ${ }^{10}$ Thus, it is of considerable value to examine if the microwave analysis could be applied to investigate the droplet size distribution of emulsified heavy oil.

Water-in-crude oil emulsion samples with water content of $10.0 \mathrm{vol} \%$ were prepared for different emulsifying time, and a representative microscope image of these samples is shown in the ESI Fig. $2 . \dagger$ It can be observed that the water phase was extremely well dispersed in crude oil. The dielectric constants of these samples were investigated by the microwave cavity, and the measured data and calculation results are presented in Fig. 8. The dielectric constant of emulsions rises slightly with the emulsifying time, while the dispersity factor increases from 0.85 at $1 \mathrm{~min}$ to 0.98 at $20 \mathrm{~min}$, indicating that the water-in-crude oil emulsions become more uniform as the emulsifying time increases.

The microwave measurements were also performed on a series of water-in-tar oil emulsion samples which were prepared for various emulsifying time. As is shown in Fig. 9, the measured dielectric constant increases with the emulsifying time, and correspondingly the dispersity factor rises considerably from 0.42 at $1 \mathrm{~min}$ to 0.70 at $20 \mathrm{~min}$. The variation of measured dielectric constant with the emulsifying time demonstrated that the microwave analysis is capable of characterizing heavy oil emulsions. 


\section{Conclusions}

We hope to have illustrated the great utility and versatility of the microwave cavity perturbation technique to provide an accurate, non-invasive method for investigating the nature of emulsions, particularly the critically important dispersion properties in various stages of the emulsifying process. The systems investigated have ranged from model emulsions to fossil fuel emulsions, illustrating the great potential of this technique.

\section{Methods}

\section{Emulsion preparation}

The emulsions were prepared utilizing a high shear homogenizer with a maximum rotation speed of $24000 \mathrm{rpm}$. A composite nonionic emulsifier was first dissolved in the oil phase, then a certain amount of deionized water was added, and shearing was performed at a fixed rotation speed. The emulsifier was a composite of Span 85 and Tween 85. In this study HLB (hydrophile-lipophile balance) $=4$ gave stable water-in-oil emulsions. The volume fraction of emulsifier was $5 \mathrm{vol} \%$ in emulsion preparation. Dodecane $(\geq 99 \%)$ and hexadecane $(\geq 99 \%)$ were purchased from Sigma Aldrich and used without further purification. Light crude oil was provided by Saudi Aramco, and coal tar was purchased from Alfa Aesar. The physical properties of the crude oil sample are shown in the ESI Table $2 . \dagger$

\section{Emulsion characterization}

Dynamic Light Scattering (DLS) was also performed immediately after the emulsion preparation to measure the droplet size distribution using a Zetasizer Nano S (Malvern Instruments). We performed each test for three times and irreversible sedimentation was not detected in such short period of measurement. A detection angle of $173^{\circ}$ was used and 10 runs were carried out in each measurement. A GXML3030 Trinocular Reflected and Transmitted Light Microscope (GT Vision) was used to observe the emulsified crude oil in this work.

\section{Conflicts of interest}

There are no conflicts to declare.

\section{Acknowledgements}

We are grateful for financial support from KACST, Saudi Arabia and EPSRC (EP/N009924/1). Yuqiang Yan thanks for a scholarship from the China Scholarship Council. We thank Professor Jason Davis for invaluable support in allowing access to the Dynamic Light Scattering equipment.

\section{References}

1 A. Lu, D. Moatsou, D. A. Longbottom and R. K. O'Reilly, Chem. Sci., 2013, 4, 965-969.
2 S. Crossley, J. Faria, M. Shen and D. E. Resasco, Science, 2010, 327, 68-72.

3 Y. Yan, L. Chen, J. Xu, M. Ren, J. Da and F. Cao, Chem. Eng. Process., 2016, 109, 90-96.

4 N. Anton, J. P. Benoit and P. Saulnier, J. Controlled Release, 2008, 128, 185-199.

5 L. D. Zarzar, V. Sresht, E. M. Sletten, J. A. Kalow, D. Blankschtein and T. M. Swager, Nature, 2015, 518, 520524.

6 E. Dickinson, Trends Food Sci. Technol., 2012, 24, 4-12.

7 T. Tadros, Emulsion Formation and Stability, John Wiley \& Sons, Incorporated, Weinheim, Germany, 2013.

8 P. K. Shivhare, A. Prabhakar and A. K. Sen, J. Micromech. Microeng., 2017, 27, 035003.

9 R. Höhler, S. Cohen-Addad and D. J. Durian, Curr. Opin. Colloid Interface Sci., 2014, 19, 242-252.

10 E. O. Fridjonsson, B. F. Graham, M. Akhfash, E. F. May and M. L. Johns, Energy Fuels, 2014, 28, 1756-1764.

11 N. N. A. Ling, A. Haber, E. F. May, E. O. Fridjonsson and M. L. Johns, Colloids Surf., A, 2014, 462, 244-251.

12 N. N. A. Ling, A. Haber, E. F. May, E. O. Fridjonsson and M. L. Johns, Chem. Eng. Sci., 2017, 160, 362-369.

13 C. Houben, G. Nurumbetov, D. Haddleton and A. A. Lapkin, Ind. Eng. Chem. Res., 2015, 54, 12867-12876.

14 S. Schuster, R. Bernewitz, G. Guthausen, J. Zapp, A. M. Greiner, K. Köhler and H. P. Schuchmann, Chem. Eng. Sci., 2012, 81, 84-90.

15 T. Hanai, Kolloid-Z., 1960, 171, 23-31.

$16 \mathrm{~J}$. C. Maxwell, A treatise on electricity and magnetism, Clarendon press, Oxford, 1881.

$17 \mathrm{~K}$. W. Wagner, Electrical Engineering (Archiv fur Elektrotechnik), 1914, vol. 2, pp. 371-387.

18 K. Lichtenecker, Phys. Z., 1931, 32, 255-260.

19 U. Erle, M. Regier, C. Persch and H. Schubert, J. Microwave Power, 2000, 35, 185-190.

20 B. R. Epstein, K. R. Foster and R. A. Mackay, J. Colloid Interface Sci., 1983, 95, 218-227.

21 J. Greffe and C. Grosse, Prog. Electromagn. Res., 1992, 6, 41100.

22 T. Zakri, J.-P. Laurent and M. Vauclin, J. Phys. D: Appl. Phys., 1998, 31, 1589.

23 B. Liu, D. R. Slocombe, J. Wang, A. Aldawsari, S. GonzalezCortes, J. Arden, V. L. Kuznetsov, H. AlMegren, M. AlKinany, T. Xiao and P. P. Edwards, Nat. Commun., 2017, 8, 514.

24 O. Klein, S. Donovan, M. Dressel and G. Grüner, Int. J. Infrared Millimeter Waves, 1993, 14, 2423-2457.

25 E. F. May, B. F. Graham, A. S. Chauhan and R. D. Trengove, Energy Fuels, 2008, 22, 3308-3316.

26 S. Carrillo De Hert and T. L. Rodgers, Chem. Eng. Sci., 2017, 167, 265-277.

27 J. A. Boxall, C. A. Koh, E. D. Sloan, A. K. Sum and D. T. Wu, Langmuir, 2012, 28, 104-110. 